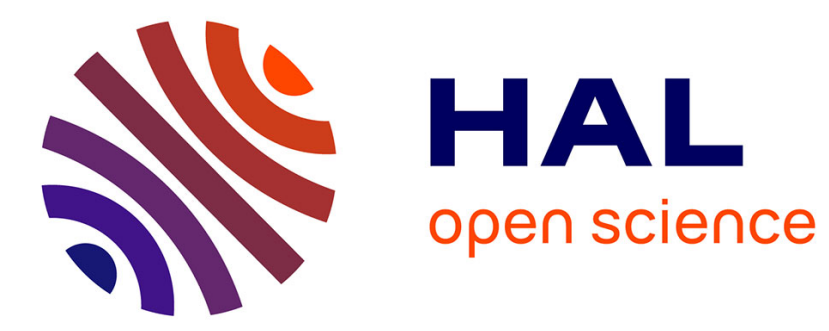

\title{
Dynamic obstacles avoidance based on image-based dynamic window approach for human-vehicle interaction
}

Yue Kang, Danilo Alves de Lima, Alessandro Corrêa Victorino

\section{To cite this version:}

Yue Kang, Danilo Alves de Lima, Alessandro Corrêa Victorino. Dynamic obstacles avoidance based on image-based dynamic window approach for human-vehicle interaction. IEEE Intelligent Vehicles Symposium (IV 2015), Jun 2015, Seoul, South Korea. pp.77 - 82, 10.1109/IVS.2015.7225666 . hal01305598

\section{HAL Id: hal-01305598 \\ https://hal.science/hal-01305598}

Submitted on 21 Apr 2016

HAL is a multi-disciplinary open access archive for the deposit and dissemination of scientific research documents, whether they are published or not. The documents may come from teaching and research institutions in France or abroad, or from public or private research centers.
L'archive ouverte pluridisciplinaire HAL, est destinée au dépôt et à la diffusion de documents scientifiques de niveau recherche, publiés ou non, émanant des établissements d'enseignement et de recherche français ou étrangers, des laboratoires publics ou privés. 


\title{
Dynamic Obstacles Avoidance Based on Image-based Dynamic Window Approach for Human-Vehicle Interaction
}

\author{
Yue Kang, Danilo Alves de Lima, Alessandro Corrêa Victorino
}

\begin{abstract}
This paper presents an approach for the development of Advanced Driving Assistance System (ADAS) based on the human-vehicle interaction using Image-based Dynamic Window Approach (IDWA). The IDWA is associated to a method for dynamic obstacles avoidance in order to prevent human driving errors, in the context of intelligent robotic vehicles. The human-vehicle interaction is presented by the correction of the Human Driving Behavior (HDB) controller for driving defaults of human drivers, with respect to referential paths that intimate the average driving path in real circumstances. The performance of the proposed human-vehicle interaction methodology, based on autonomous embedded functionalities, is simulated and verified in different bypass scenarios.

Index Terms - Keywords: Obstacle avoidance, Image-based Dynamic Window Approach, human-vehicle interaction, driving safety, parameter regulation.
\end{abstract}

\section{INTRODUCTION}

Traditional Advanced Driver Assistance Systems (ADAS) have been advanced with a considerably fast pace ever since its first appearance. Lateral and longitudinal stabilization and control, careful active actions and warnings and full active actions build up the three steps of development for ADAS [1], respectively represented by the widely renowned Anti-lock Braking System (ABS), the Adaptive Cruise Control (ACC) [2] and more recently the Collisions Avoidance (CAV) by emergency braking [3]. Different approaches from various directions of research contribute to the core interest of this field: driving safety [4].

It has been accepted without doute that safety is of critical interest to the intelligent transportation systems. Supervision, control and correction of human driving characteristics have long been a major issue to the driving safety on the road [5]. A great deal of researchers devote their effort into the modelling of human driving behaviors for the purpose of predicting and correcting such potential danger introduced by humankind. For instance, [6] proposed a safety-based approaching behavioural model for the driver's car-following behavior with various driving characteristics. A more detailed model of driving behavior characteristics analysis is illustrated in [7] with a weight determination based on influences of various driving behaviors on conflict. More recently, a novel method for predicting the next contextual changing point of driving behavior on the basis of a Bayesian double articulation analyzer was implemented in [8].

\footnotetext{
The authors are with Heudiasyc UMR CNRS 7253 Université de Technologie de Compiègne. This work is founded in part by the VERVE Project (Picardie region and Europe), in the context of the MS2T LABEX and ROBOTEX projects. Yue Kang holds a Ph.D scholarship from Chinese Scolarship Council and Picardie region in France. Contact authors yue.kang@hds.utc.fr.
}

The continuous development in such field supports the point that, while fully autonomous intelligent vehicles remains currently limited by critical circumstance and enriched database [9], research that focuses on improving current driving systems for human drivers is still of irreplaceable significance [3]. Human-vehicle interaction is therefore emphasized in the past years and decades for the purpose of decreasing the hazard of human factors in driving circumstance. Primitive modelings and simulations could date back to [10] and [11] for instance, followed by a seriel of approaches from different directions of research [12] [13]. Placing emphasis on human-vehicle interaction, as a bridge between human drivers and safety-supporting assistance systems, obviously meets the needs of safe diving.

As a complement of traditional ADAS, as well as a combination of human driving behaviors modelling, our initial approaches [14] and [15] aimed at simulating and correcting the human drivers' decision faults in humanvehicle interaction accomplished by applying the Imagebased Dynamic Window Approach (IDWA) [16], a variation from the original DWA [17], using the visual information of road land and center provided by a pinhole camera, with an implement of obstacle detection and avoidance guaranteed by an occupancy grid [18],. The controlling faults by human drivers were presented as failures during static obstacles avoidance. In this paper, we march forward in the same direction of research by replacing the static obstacles in the scenario with dynamic, "moving" ones, for the purpose of a better representation of real driving circumstances.

This article is organized as follows: in Section II, we present the robot model and the simulation environment; in Section III, we present the fondamental concepts of the DWA algorithm, the design of human-vehicle interaction, the $\mathrm{Hu}$ man Driver Behavior (HDB) controller and the configuration of dynamic obstacles; experimental results and performance analysis are presented in Section IV.

\section{General Definitions}

As a consequence of our previous work [14] [15], we continue our research and validation by preserving the robotic model that was implemented in the initial experimental environment. Without special indication, we do not particularly distinguish between the following conceptions: "the robot", "the robotic model" and "the (experimental) vehicle". The robot follows the path according to the kinematic model of 


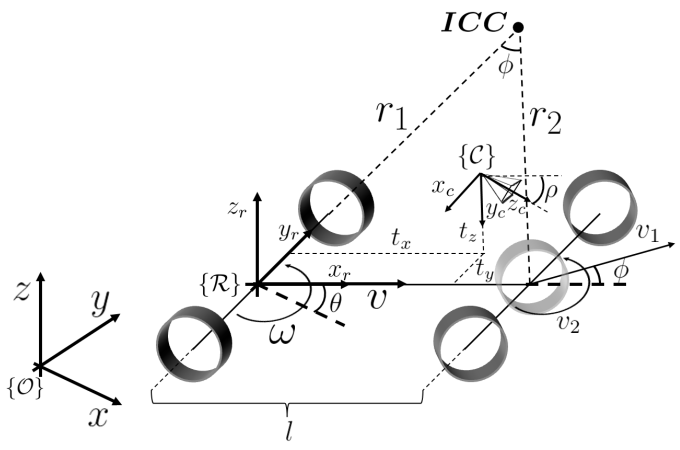

Fig. 1. Kinematic model diagram for a front wheel car-like robot. In this model the vehicle reference frame $\mathcal{R}$ performs circular trajectories related to the instantaneous center of curvature (ICC).

a front wheel car [19] as:

$$
\left[\begin{array}{c}
\dot{x_{r}} \\
\dot{y_{r}} \\
\dot{\theta} \\
\dot{\phi}
\end{array}\right]=\left[\begin{array}{c}
\cos \theta \cos \phi \\
\sin \theta \cos \phi \\
\sin \phi / l \\
0
\end{array}\right] v_{1}+\left[\begin{array}{l}
0 \\
0 \\
0 \\
1
\end{array}\right] v_{2},
$$

where the vehicle configuration is given by $q=\left[x_{r} y_{r} \theta \phi\right]^{T}$, with the position $\left(x_{r}, y_{r}\right)$ and orientation $(\theta)$ of the car's reference frame $\{\mathcal{R}\}$ in relation to a static world reference frame $\{\mathcal{O}\}$, and $\phi$ is the average steering angle of the front wheels by the Ackerman's approximation. The orientation and steering angles $(\theta$ and $\phi)$ are positive counter-clockwise, with $\theta \in]-\pi, \pi]$ and $\phi \in\left[-\phi_{\max }, \phi_{\max }\right]$. The variables are illustrated in the Figure 1.

The control input for the vehicle of the model (1) is $u=$ $\left[\begin{array}{ll}v_{1} & v_{2}\end{array}\right]^{T}$, which consists of respectively the linear velocity $v_{1}$ and the steering velocity $v_{2}$ of the front wheels. With the relationship between linear velocity $v$ and front wheels velocity $v_{1}$ as $v=v_{1} \cos \phi$, as well as the angular velocity $\dot{\theta}=v_{1} \cos \phi / r_{1}=\omega$ and the steering angle, it is possible to choose the control input for the robot as $u_{r}=[v \omega]^{T}$. The robot in our work is considered to be set in a planar road. In order to focus our research on the algorithms of avoiding dynamic obstacles, we simplify the environment of simulation as a straight road with two driving lanes. The width of the lane and the size of the vehicle are proportional to the real driving circumstances. A typical example schema of the simulation environment in global version is illustrated in Figure 2(a). The visual information utilized for the IDWA algorithm is provided by a pinhole camera. The camera frame $\{\mathcal{C}\}$ is also represented in Figure 1 with optical center position in $\left(x_{c}, y_{c}, z_{c}\right)=\left(t_{x}, t_{y}, t_{z}\right)$ in the robot frame and a constant tilt offset $0<\rho<\frac{\pi}{2}$ related to the $x_{r}$ axis. Figure 2(b) shows a typical example of the image captured by the camera, from which the information of the road lane center is calculated and provided to IDWA as the referencial of the vehicle's heading.

Compared with our previous work, the modification to the obstacles is of the most significance in this article. The obstacles, previously represented by static blocks with similar size to our vehicle, are currently enhanced with the

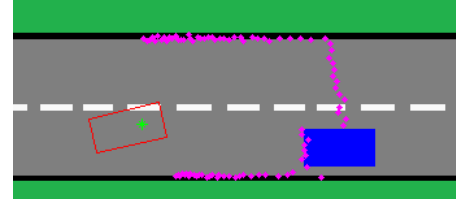

(a) Example of the global version during a bypass procedure.

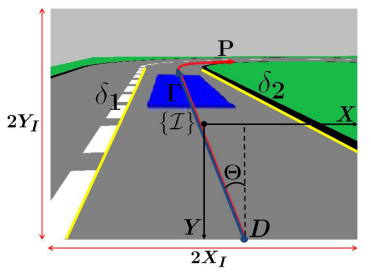

(b) Example of the cameral version.

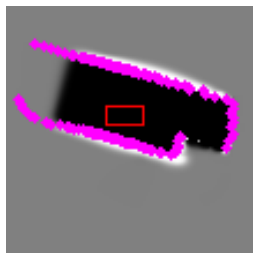

(c) Vehicle center oriented occupancy grid.
Fig. 2. Typical schema of the environment of simulation.

identical kinematic model given by the equation (1). This modification enables the capacity of displacement to the obstacles, while maintaining a relatively simple controlling manoeuvre: similar control strategies of the vehicle can be applied to the obstacles to simulate real driving circumstances on the road.

\section{Dynamic Obstacles Avoidance}

\section{A. Image-based Dynamic Window Approach}

Recalling the fondamental algorithm of obstacle avoidance of our previous work [14], we present a brief introduction of the Imaged-based Dynamic Window Approach (IDWA), which is a reactive technique in obstacle detection and avoidance, originally proposed by [17] and advanced by [16] To accomplish the propose of detecting and bypassing the obstacles, an objective function (2) is optimized in order to select the best control input regarding the desired configuration to the robot. The objective function is constituted with three weighted components: the goal position (heading), the obstacle distance (dist) and the final linear velocity (velocity), represented as:

$$
\begin{aligned}
D W A(v, \omega)= & \alpha \cdot \operatorname{heading}(v, \omega)+\beta \cdot \operatorname{dist}(v, \omega) \\
& +\gamma \cdot \operatorname{velocity}(v, \omega) .
\end{aligned}
$$

As a consequence of the optimisation, the dynamic window search space, which considers the actual speed of the vehicle, its accelerations, the obstacles in the workspace, and also the physical limits of the vehicle is consequently generated as:

$$
V_{D W}=V_{d} \cap V_{a} \cap V_{s} .
$$

where $V_{d}$ is the set of all reachable velocities in a time interval $\Delta t$ according to the current linear and angular acceleration of the vehicle, $V_{a}$ represents the admissible velocities classified by the distance to the obstacles, and $V_{s}$ is the set of velocities that satisfy the constraints of the maximum accelerations. In this proposed approach of human-vehicle interaction, the driver is considered to provide 
an input $\left[\begin{array}{ll}v & \omega\end{array}\right]^{T}$ for the driving control, which is then delivered to the IDWA navigation method for the verification (and correction if needed).

The progress of IDWA from the original DWA is the application of the visual information, which is provided by the camera implemented on the vehicle. From the image captured by the camera(Figure 1), the road lane as well as its center is obtained. Consequently, the road center information builds up a precise goal position (heading) for the objective function (2).

For the purpose of better utilizing the algorithm of IDWA, a process of the regulation of the three weighting parameters $\alpha, \beta$ and $\gamma$ in (2) is of apparent significance. Noticing that the three parameters possess an obvious similarity to those of the widely known PID controller, we implement a simple tuning method, during which we simplify the procedure of determining the absolute values by normalizing the output of the objective function (2) after applying the pre-tuned parameters, as the order of magnitude of the parameters holds a prior significance to the absolute values.

In addition, while the original DWA algorithm does not include the manoeuvre of obstacle detection, we implement the algorithm with a LIDAR sensor [16]. The sensor provides the support of obstacle detection by establishing an occupancy grid [18] with real-time update, as is shown in Figure 2(c). The occupancy grid provides a "drivable" zone for the vehicle, i.e. a limitation for the proper function of DWA.

\section{B. Human-vehicle interaction}

The interaction between human drivers and the vehicle in our approach is represented by the correction of potentially dangerous behaviors due to a lack of proper reaction(deceleration, turning, etc.) when engaging the obstacles. During the simulation, the decision of the driver is expressed by a referential path for the controller, for the intention of reducing the time cost of calculation. An example of referential paths of human driver behaviors is shown in Figure 3, in which an overtaking behavior is represented in Figure 3(a), and a fault driving behavior is represented in Figure 3(b). It is important to note that these referential paths are not planned trajectories to be followed by the autonomous vehicle, but a mathematical representation of possible behaviors of the human drivers, which are to be corrected or optimized by the intelligent navigation systems (based on the IDWA).

The design of the controller to our vehicle is also derived from our previous work [14] and [15], which is proved to function adequately for the propose of representing the primary driving behavior of a human driver. Given that human drivers tend to keep the vehicles in the center of the road lane, we establish a referential path by assembling the coordinates $\left(x_{p}, y_{p}\right)$ of the virtual middle line of the drivable lane. Based on the referential path coordinates, the design of the Human Driving Behavior (HDB) controller is represented as:

$$
\omega=\frac{v_{d}}{v_{l}} \sqrt{\left(x_{r}-x_{p}\right)^{2}+\left(y_{r}-y_{p}\right)^{2}}+\beta,
$$

where $\omega$ is the feedback gain of the controller, $v_{d}$ is the desired linear velocity of the tracking control, $v_{l}$ is the velocity limitation of the vehicle, $\left(x_{r}, y_{r}\right)$ are coordinates of the position of the vehicle, $\left(x_{p}, y_{p}\right)$ are coordinates of the corresponding point on the referential path, and $\beta$ is an additive white Gaussian noise (AWGN) which represents inaccuracy of human drivers' control.

As human drivers rarely have access to the precise drift angle of the vehicle, the control law does not involve any direct angular information. Instead, the controller functions as a negative feedback regulator, similar to the proportional section of a classic PID controller, according to the error or the deviation between the position of the vehicle and the decided path.

\section{Dynamic obstacles}

The obstacles in our approach are considered to be moving vehicles on the road with inferior linear velocity, with which an overtaking determination by the following car is essential for the purpose of avoiding the collision. The visual outcome of an obstacle is identical to that in our initial work: a blue opaque planar rectangle with the same size of the experimental vehicle (the Figure 2(a)), due to the fact that the detection of obstacles in our work is accomplished by a simulative algorithm of 2D LIDAR.

To imitate the actual driving circumstances, we apply the same kinematic model (1) to the car-like obstacles, with limited linear velocity and basic control algorithm. An obstaclebehaved vehicle forwards as well in the middle of the road lane, without direct communication to our experimental vehicle or other obstacle vehicles. In a circumstance with multiple obstacle vehicles, the velocities of each vehicle in the same direction are set differently in order to avoid the collision or overlapping.

Since the obstacles become "moving" in the scenario, the previous method of evasive trajectory [3] in our initial work (Figure 3(a)) is no longer suitable, as it requires a static off-line calculation before being applied to the avoidance procedure. However, we observe in [14] that the design of HDB controller is capable to handle a predefined referential path that intersects or directly goes across obstacles, which is considered as a representative danger behavior in driving and is possible to be corrected by HDB+IDWA control pattern. We therefore utilize an ensemble of the coordinates $\left(x_{p}, y_{p}\right)$ of the virtual middle line of the drivable lane as the referential path for the vehicle, as is shown in the Figure 3(b). Since the set of admissible control inputs $V_{D W}$ is calculated in each iteration of control, the correction of human drivers' faults is therefore guaranteed, as well as the security of the driving tasks.

\section{EXPERIMENTAL RESULTS}

The experimental simulation is proceeded on Matlab R2014a (8.3.0.532). The environment consists of an open track (7.8 meters in width) with different quantity of obstacles ( 5.86 by 2.92 meters each). The ratio of the image is 0.0630 meter per pixel. 


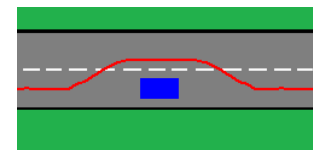

(a) Initial evasive trajectory (b) Referential path applied for static obstacle avoidance. in dynamic obstacle avoidance.

Fig. 3. Revision of the referential path.

\section{A. Tuning of the parameters of IDWA}

Recall the objective function of IDWA:

$$
\begin{aligned}
D W A(v, \omega)= & \alpha \cdot \operatorname{heading}(v, \omega)+\beta \cdot \operatorname{dist}(v, \omega) \\
& +\gamma \cdot \operatorname{velocity}(v, \omega) .
\end{aligned}
$$

The first section of our experiment aims at the effect of different tunings of the parameters in the objective function (5), namely $\alpha, \beta$ and $\gamma$, representing respectively the goal position (heading), the obstacle distance (dist) and the final linear velocity (velocity). Given that the determinant of the parameters relies on their order of magnitude rather that their absolute values, we regulate the parameters with a stepping rate of 5 , in other words half of one order of magnitude.

For the purpose of a better regulation of the parameters, each experimental simulation starts with idetical initial condition: the scenario consists of one single dynamic obstacle; the vehicle starts with the same linear velocity $(5 \mathrm{~m} / \mathrm{s}$, twice of the velocity of the obstacle) and angular velocity (zero); the initial position of the obstacle is outside of the detection of LIDAR lasers.

Table I illustrated the results of bypass time in different

TABLE I

BYPASS TIME IN DIFFERENT PARAMETER SETTINGS

\begin{tabular}{c||c|c|c|c|c|c}
$\gamma \beta$ & $0.1 \alpha$ & $0.5 \alpha$ & $\alpha$ & $5 \alpha$ & $10 \alpha$ & $50 \alpha$ \\
\hline \hline $0.1 \alpha$ & N/A & N/A & N/A & N/A & $\mathrm{X}$ & $\mathrm{X}$ \\
\hline $0.5 \alpha$ & N/A & N/A & N/A & N/A & $\mathrm{X}$ & $\mathrm{X}$ \\
\hline$\alpha$ & N/A & N/A & $\mathrm{X}$ & $\mathrm{X}$ & 27.1 & 23.2 \\
\hline $5 \alpha$ & N/A & N/A & $\mathrm{X}$ & 22.1 & 20.3 & 19.1 \\
\hline $10 \alpha$ & N/A & $\mathrm{X}$ & 19.2 & 17.4 & 15.7 & 15.2 \\
\hline $50 \alpha$ & $\mathrm{X}$ & 19.4 & 17.9 & 15.2 & 14.8 & 14.6 \\
\hline
\end{tabular}

Unit: $\mathrm{s}$

parameter settings. "Bypass time" is defined as the time interval which begins from the moment the obstacle is detected by the laser sensor, and ends at the moment the robot establishes a stable linear velocity after returning the original lane. The " $\mathrm{X}$ " marks in the Table I indicated that, with such regulation of the parameters, the robot failed to behave the bypass procedure for at least once during the experiment; in other words the vehicle followed the obstacle in front and did not overtake it. The "N/A" marks represented the omission of such combination of parameters during the simulation.

The results indicated the emphasis on the obstacle distance (dist) and the final linear velocity (velocity) terms in the objective function 5, compared with the goal position (heading) term. The vehicle appeared more likely to perform a low-speed following behavior when the parameter $\alpha$ was relatively large, for the reason that the IDWA algorithm tends to hand out a result with which the vehicle maintains its heading with respect to the referential path. Such appearance indicates that, should emphasis be put on heading, the human-vehicle interaction functions in a greater degree as a limitation of the overtaking behavior.

On the other hand, with the augmentation of the parameters $\beta$ and $\gamma$, the bypass behavior of the robot was gradually and reliably guaranteed, and the time cost of overtaking was also reduced. The human-vehicle interaction under such preference appear to enable the overtaking behavior, which is regarded as a correction performed by the system when the linear velocity of the vehicle is faster than the obstacles ahead.

However, we observed that the reduction of bypass time was obviously slowed down when both $\beta$ and $\gamma$ reached the ratio of 50 to the value of $\alpha$. We conclude that, alongside with the augmentation of $\beta$ and $\gamma$, IDWA calculation aggressively puts emphasis on maintaining a relatively high linear velocity and keeping the furthermost distance between the vehicle and the obstacles, thus neglecting the guidance of the vehicle to return back to the original lane. Consequently, we regulate the parameters as $\beta=\gamma=50 \alpha$ at the moment.

\section{B. Validation of dynamic obstacles avoidance}

In order to validate our approach of dynamic obstacles avoidance implemented by the HDB+IDWA control pattern, as well as the tuning of the parameters in Section IV-A, we complicate the scenario of driving by increasing the number of the dynamic obstacles and their linear velocities. The first step consists of two dynamic obstacles in the same direction of the robot, the one in the front possessing a higher linear velocity in order to avoid the collision between the obstacles themselves.

The result in the Figure 4 illustrated that the configuration
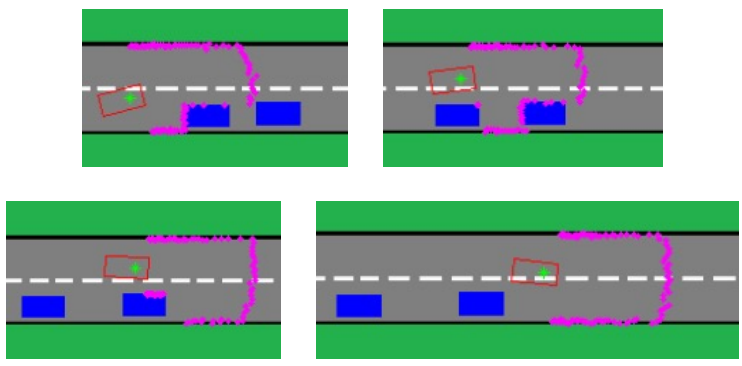

Fig. 4. Validation of dynamic obstacles avoidance.

of HDB+IDWA control pattern in Section IV-A is capable to ensure the avoidance of a sequence of several dynamic obstacles in the same direction. As long as the linear velocity of the robot and distance from the obstacles are guaranteed by IDWA algorithm, the bypass process remains smooth and secured, as is shown in the Figure 5. 


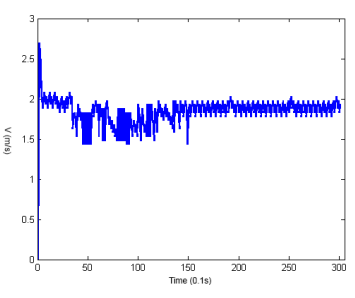

(a) Linear Velocity.

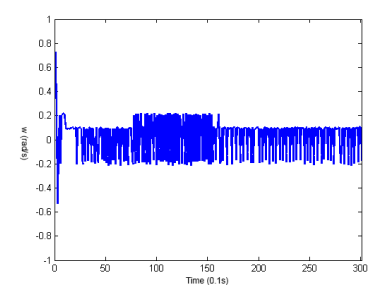

(b) Angular Velocity.
Fig. 5. Velocities of the robot during the bypass validation.

To imitate the real driving condition, we complicate furthermore the scenario by moving one of the dynamic obstacles on the opposite lane with reversed direction. The newly introduced obstacle initially blocks the possible bypass path of the robot until it leaves the detection zone of the LIDAR sensor. Hence, the robot is forced to perform a car-following behavior before the possibility of safe bypass is ensured.

Figure 6 illustrated the "follow-and-bypass" procedure of

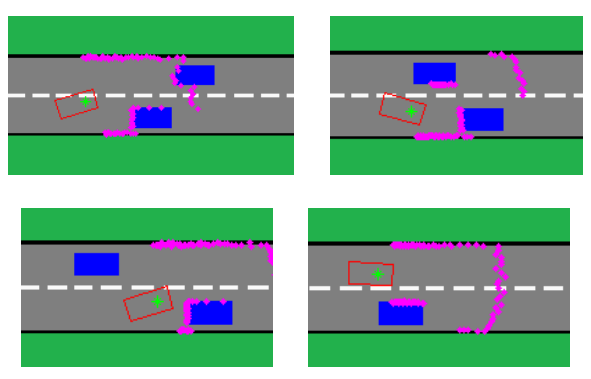

Fig. 6. Obstacle avoidance by "follow-and-bypass" behavior.

the vehicle engaging the dynamic obstacle in the opposite direction. The vehicle followed the car in front with a relatively low linear velocity (Figure $7(a)$ ) to ensure the driving safety, guaranteed by the obstacle distance (dist) term and its parameter $\beta$. As a consequence, the bypass time interval of the second obstacle was prolonged, due to the need for the vehicle to restore its linear velocity.

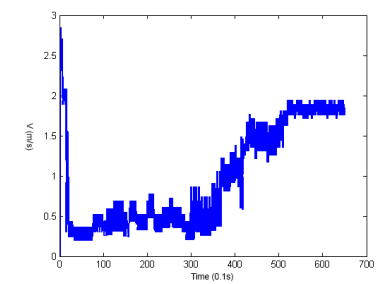

(a) Linear Velocity.

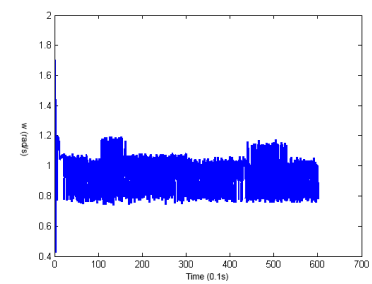

(b) Angular Velocity.
Fig. 7. Velocities of the robot during the "follow-and-bypass" procedure.

An important remark is that, during this simulation, the bypass procedure was not always ensured, as the vehicle continued following the obstacle in front with a low linear velocity. In addition, we observed that in several situations the vehicle moved so close to the road lane (for the purpose to keep the distance to the opposite obstacle) that the IDWA algorithm determined that no available linear velocity could be verified, not to mention provided. This deficiency is noteworthy as it limits the practicality of the proposed method.

\section{CONCLUSion}

In this paper we have presented an approach of dynamic obstacle avoidance for car-like robot, with the help of IDWA algorithm and HDB controller based on our previous work. The results of the experimental simulation have proved that such control manoeuvre is capable to ensure the driving safety while performing the avoidance of dynamic obstacles.

The regulation of the three parameters in the objective function of IDWA algorithm have revealed the significance of the obstacle distance (dist) and the final linear velocity (velocity) criteria during the dynamic obstacle avoidance. While focusing on the bypass procedure, these two terms hold the prior focus to the heading of the vehicle. This phenomenon is comprehensible as the avoidance of the obstacles calls for migrations from the referential path of driving.

The deficiency of this approach is mainly the issue of relatively low linear velocity of the vehicles, both the robot and the dynamic obstacles. The limitation is generally due to the usage of kinematic model of the vehicle, which provides a relatively inaccurate fundament for the simulations. Improvement towards such defect is promised to optimize the practicability of this approach in real driving circumstances.

Another direction of improving our approach lies in variable parameters for the IDWA algorithm. A real-time, self-adaptive and intelligent method for the regulation of the parameters is believed to be more than profitable, as the system will be more capable to handle with different circumstances, thus providing a higher level of security of driving.

This paper has illustrated the progress in our series of work in the project VERVE ${ }^{1}$. Our future research will proceed particularly in the experimental platform of SCANeR ${ }^{\mathrm{TM}}$ Studio, before being finalized on the experimental vehicles for the validation of the methodology.

\section{REFERENCES}

[1] S. Borhade, M. Shah, P. Jadhav, D. Rajurkar, and A. Bhor, "Advanced driver assistance system," in Sensing Technology (ICST), 2012 Sixth International Conference on. IEEE, 2012, pp. 718-722.

[2] A. Vahidi and A. Eskandarian, "Research advances in intelligent collision avoidance and adaptive cruise control," Intelligent Transportation Systems, IEEE Transactions on, vol. 4, no. 3, pp. 143-153, 2003.

[3] R. Isermann, R. Mannale, and K. Schmitt, "Collision-avoidance systems proreta: Situation analysis and intervention control," Control Engineering Practice, 2012.

[4] A. Tang and A. Yip, "Collision avoidance timing analysis of dsrcbased vehicles," Accident Analysis \& Prevention, vol. 42, no. 1, pp. $182-195,2010$

${ }^{1}$ The project VERVE stands for Novel Vehicle Dynamics Control Technique for Enhancing Active Safety and Range Extension of Intelligent Electric Vehicles. 
[5] F. Lotz, "System architectures for automated vehicle guidance concepts," in Automotive Systems Engineering. Springer, 2013, pp. 3961.

[6] W. Wang, W. Zhang, H. Guo, H. Bubb, and K. Ikeuchi, "A safety-based approaching behavioural model with various driving characteristics," Transportation research part C: emerging technologies, vol. 19, no. 6, pp. 1202-1214, 2011.

[7] X. Ge, J. Lu, Q.-J. Xiang, and P.-Y. Wang, "Study on driving behavior and traffic conflict at highway intersection," in Power Electronics and Intelligent Transportation System, 2008. PEITS '08. Workshop on, 2008, pp. 492-495.

[8] S. Nagasaka, T. Taniguchi, K. Hitomi, K. Takenaka, and T. Bando, "Prediction of next contextual changing point of driving behavior using unsupervised bayesian double articulation analyzer," in Intelligent Vehicles Symposium Proceedings, 2014 IEEE. IEEE, 2014, pp. 924931.

[9] A. Houenou, P. Bonnifait, V. Cherfaoui, and W. Yao, "Vehicle trajectory prediction based on motion model and maneuver recognition," in Intelligent Robots and Systems (IROS), 2013 IEEE/RSJ International Conference on, 2013, pp. 4363-4369.

[10] R. W. Allen, T. J. Rosenthal, and J. R. Hogue, "Modeling and simulation of driver/vehicle interaction," SAE Technical Paper, Tech. Rep., 1996.

[11] M. Kopf and G. Nirschl, "Driver-vehicle interaction while driving with acc in borderline situations," in MOBILITY FOR EVERYONE. 4TH WORLD CONGRESS ON INTELLIGENT TRANSPORT SYSTEMS, 21-24 OCTOBER 1997, BERLIN.(PAPER NO. 2128), 1997.

[12] C. Harvey, N. A. Stanton, C. A. Pickering, M. McDonald, and P. Zheng, "In-vehicle information systems to meet the needs of drivers," Intl. Journal of Human-Computer Interaction, vol. 27, no. 6, pp. 505-522, 2011.

[13] A. L. Kun, A. Schmidt, A. Dey, and S. Boll, "Automotive user interfaces and interactive applications in the car," Personal and Ubiquitous Computing, pp. 1-2, 2013.

[14] Y. Kang, D. A. de Lima, and A. C. Victorino, "An approach of human driving behavior correction based on dynamic window approach," in Intelligent Vehicles Symposium Proceedings, 2014 IEEE. IEEE, 2014, pp. 304-309.

[15] Y. Kang, D. A. de Lima, and A. Correa Victorino, "Driving behavior correction in human-vehicle interaction based on dynamic window approach," in Cyber Technology in Automation, Control, and Intelligent Systems (CYBER), 2014 IEEE 4th Annual International Conference on. IEEE, 2014, pp. 485-490.

[16] D. A. Lima and A. C. Victorino, "An image based dynamic window approach for local navigation of an autonomous vehicle in urban environments," in Modelling, Estimation, Perception and Control of All Terrain Mobile Robots (WMEPC), 2014 IEEE ICRA Workshop on, Juin 2014, pp. 120-125.

[17] D. Fox, W. Burgard, and S. Thrun, "The dynamic window approach to collision avoidance," Robotics \& Automation Magazine, IEEE, vol. 4, no. 1, pp. 23-33, 1997.

[18] A. Elfes, "Using occupancy grids for mobile robot perception and navigation," Computer, vol. 22, no. 6, pp. 46-57, 1989.

[19] A. D. Luca, G. Oriolo, A. De, and C. Samson, Robot Motion Planning and Control. Springer Berlin / Heidelberg, 1998, vol. 229, ch. Feedback Control Of A Nonholonomic Car-Like Robot, pp. 171-253. 